\title{
Lessons Learned in Deploying Independent Living Technologies to Older Adults' Homes
}

\author{
Julie Doyle*, Catherine Bailey^ ${ }^{\wedge}$ Cliodhna Ni Scanaill ${ }^{\circ}$, Flip van den Berg§ \\ *CASALA, Dundalk Institute of Technology, Dublin Rd., Dundalk, Co. Louth, Ireland \\ ${ }^{\wedge}$ School of Health Community and Education Studies, Northumbria University, UK \\ ${ }^{\circ}$ Intel Digital Health, Intel Ireland, Leixlip, Co. Kildare, Ireland \\ $\S$ TRIL Centre, University College Dublin, Dublin 4, Ireland
}

Corresponding author information:

Julie Doyle

CASALA, PJ Carrolls Building, Dundalk Institute of Technology, Dublin Rd., Dundalk, Co. Louth, Ireland

E: Julie.doyle@,casala.ie

P: $+353(0) 429370497$

\begin{abstract}
Independent living technologies are fast gaining interest within both academia and industry, amid the realisation that the world's population is ageing. Technology can increase the quality of life of older people, allowing them to age in-place and helping them to remain physically, cognitively and socially engaged with their environment. However, little research in this area is applied. We argue the necessity of moving such technology out of the research lab and into the home, where its real impact on the lives of older adults can be assessed. This paper outlines a series of recommendations encompassing the life cycle of independent living technologies, from ethnographic assessment, through to design, deployment and evaluation. This work is based on lessons we have learned deploying such technologies to older people in over 200 homes. We believe this paper can act as a guide for other researchers interested in developing technologies with older people.
\end{abstract}

Keywords

Independent living technologies, Older adults, Recommendations, Ethnography, Design, Deployment, Evaluation. 


\section{INTRODUCTION}

Population projections estimate a significant increase in the number of older adults in the near future [21, 24]. By 2050 an estimated $22 \%$ of the world's population - nearly 2 billion people - will be aged 60 or over [36]. This will result in a shortage of workers and facilities to care for the ageing population. Thus, improving the period of health ageing, by enabling older adults to maintain an active role in society and to manage their own health in the place of their choice, is an essential and pressing need. While for many ageing is a positive experience, dependency in this cohort will present major economic and social challenges. Advances in technology have enabled healthcare information and communication technologies (ICT) to become increasingly pervasive, moving from controlled clinical environments to real homes. As a result, many academic and industrial initiatives have been launched to promote the design and development of ICT, which monitors health, increases mobility, facilitates social connection and enhances cognitive function $[1,8,17]$ These independent living technologies may enable older adults to live independently in the place of their choice as they age, and ultimately increase their quality of life and wellbeing, in addition to reducing the cost of healthcare.

Much progress has been made in recent years in developing technology interventions to support ageing in place $[1,23]$. These include ambient assisted living technologies [30], technologies to monitor activities of daily living [5], health management systems [26], and more interactive 'wellness' technologies such as applications delivered through interactive TV [8] or standalone devices $[16,17]$ to name but a few.

However, there are many complexities in designing, developing, deploying and evaluating independent living technologies for older adults. Existing research and guidelines on these topics usually have a rather limited focus, such as interface design, the user-centred design process and the development of usable technology for older adults [20,37]. While these are all critical to the overall design and development of such technologies, comparatively little research focuses on the wider challenges of deploying them to older adults in their homes. Those that do tend to focus on only one aspect. For example, Dickinson et al. describe the importance of the requirements gathering process occurring in the home [13]. Rodriguez-Ascaso et al. [29] present a number of guidelines for home telecare services, based on a review of scientific and technical literature, rather than experiences of deploying such technologies. Their guidelines are organised into categories including user's trust, user interaction and service aspects.

In this paper, we reflect on these complexities and outline a series of recommendations for the deployment of independent living technologies into the homes of older adults. We base our recommendations around an analytical exploration of lessons learned from multiple home visits and participatory design sessions with older adults across a number of projects we have worked on. We provide 2 case studies to illustrate these recommendations; the first a small form-factor, standalone device, used to enhance awareness of alertness; and the second a large PC and sensor-based installation, developed to deliver an interactive exercise programme. We discuss the common and specific difficulties experienced in designing, deploying, and uninstalling both these independent living technologies into the homes of older adults. We also draw from the existing literature, 
particularly on the importance of a participatory design process in developing independent living technologies. A vast amount of information exists on the characteristics and needs of older adults and much of this has been used in the design process of ICT for older people. Where the literature is lacking, however, is solutions to address the challenges of actually deploying these technologies to the homes of older people and ensuring their seamless integration into their day-to-day lives. Furthermore, in academic research it is often young, early-stage researchers and not more senior researchers, who conduct research in the field. We believe that the recommendations we propose will help such researchers to be more successful when working with older adults.

\section{CHALLENGES IN DESIGNING HOME-BASED TECHNOLOGIES FOR OLDER ADULTS}

Independent living technologies should assist older adults in their day-to-day lives. However, technology can often represent a barrier for older adults, presenting a major challenge to learn and use, rather than acting as a facilitator. There are many reasons for this including unfamiliarity, computer anxiety and inaccessible technology [10]. Furthermore, cognitive disabilities resulting from age degenerative processes can significantly increase the learning curve of older adults, making it more difficult and time consuming for this group to learn new skills, compared to younger adults. Physical impairments related to sensory loss are another obvious effect of ageing [20]. Such impairments affect visual, auditory and tactile capabilities, further distancing older adults from technology. Sainz Salces et al [30] provide a detailed discussion on the physical and cognitive effects of ageing. The above-mentioned factors are not only important in designing a usable system for older adults. They also affect whether or not an older adult might want to use such technology. While it is generally agreed that older people are capable of learning new skills [19], as noted in [27] the effort required to learn something new may be perceived as not worth the trouble for the expected gain. Therefore, it is important to understand what might motivate older people to want to use such technology. Designers of applications that target older adults as a user group must understand this cohort's attitudes towards technology and communication and ensure applications are designed with their unique needs in mind.

A further challenge of designing for older peoples' homes is to ensure technologies are not stigmatising. Sokoler and Svensson describe non-stigmatising technology as "technology that can be used without putting older people on display as disabled, needy or weak" [33]. Not only might older adults not want stigmatising technologies on display within their home, they may also have a negative impact on the individual, heightening their awareness of their health issues and signifying a need to be monitored. Such issues can have a significant impact on the ongoing use of a technology.

Despite these challenges, our research like others [12] has found that older adults are not averse to new technology, providing that it is useful, practical and appropriately introduced. For example, older people need space to be curious about a new technology, to feel comfortable to ask what they may think are 'stupid' questions 
and have these answered, and eventually replacing understandable worry about doing something wrong, with a building confidence and excitement about the potential of the technology.

Our own research has taught us that moving technologies out of the lab and into real homes presents another set of challenges. Technologies to be deployed to homes should not only be aesthetically pleasing; they should also unobtrusively integrate with existing household contents, helping to ensure the home owner will not want to hide the technology away. It is thus necessary to work with the home owner to decide on the best placement of technology. Here we have to ensure not only that the technology is placed in a location suitable for effective data collection, but also that the home-owner is comfortable with its placement. Not getting this right might mean the technology is not adopted.

There are further challenges associated with evaluating the impact and usability of technologies in the home. Methods are required for the effective collection of quantitative and qualitative data, without observers or evaluators being present in the home, enabling researchers to make informed design decisions and to optimise the user experience. Real homes are different to smart homes - they are different shapes and sizes and may contain potential obstacles to home deployments. Real homes have not been designed to integrate specific technologies. Therefore, there are practical concerns such as access to cabling, power sources and Internet connectivity and these need to be addressed before any technology is deployed. There may also be existing technologies in the home that will need to be integrated with any new technologies being introduced, for example a programme that works through the person's television. A major challenge is understanding the complexity of how a person uses their living space, including their routines, movements and activities.

These unique challenges, which we and others have faced in the design of technologies for older people, have motivated the writing of this article. In the following section we describe two case studies to illustrate the types of projects we have implemented, setting the scene for the recommendations we outline in the remainder of the article.

\section{THE CASE STUDIES}

The case studies below describe two interactive independent living technologies deployed by the Technology Research for Independent Living (TRIL) Centre, into the homes of older Irish adults. TRIL is an active collaboration between researchers in academic, clinical and industry settings. The centre harnesses multidisciplinary ageing research, clinical expertise and enabling technology development and evaluation to support independent living. Research and innovation in the TRIL Centre is underpinned by a design-ethnography ${ }^{1}$ process that facilitates the development of research prototypes leading to evaluations with older people in their own homes. The goal is to better understand how technology can enable health and social care in the community

\footnotetext{
${ }^{1}$ Design-ethnography is a set of methods towards the goal of understanding people in order to better understand their lives. Design ethnographers have the opportunity and skills to run with their insights and make solutions.
} 
and home. These case studies describe two very different technologies, developed using the TRIL participant-led cycle of technology innovation for two different research themes; cognitive decline and falls prevention. This section provides an overview of these projects, which will be referred to throughout the paper to provide context for our experiences and recommendations.

\section{Case Study One: Alertness - Training for Focused Living}

The "Alertness: Training for Focused Living" training project aims to raise awareness around the concepts of alertness and attention, and their importance in our everyday lives. The Alertness Training Programme is a fourweek, self administered home-based training programme, which teaches older adults a technique to modify their alertness at will. Participants receive an Alertness Training Kit in the mail, consisting of the biofeedback device and a guidebook that provides education on alertness, reflective questions and instructions for the self-alerting technique and biofeedback device. Participants are encouraged to work through the guidebook 5 days per week. This study uses galvanic skin response (GSR) biofeedback as a tool to enhance the user's learning of the selfalerting technique. The form factor - a cushion-like design - prevents the device from appearing medical or technical (Fig.1). Therefore it is more approachable to non-technically inclined older adults. GSR data is captured onto an in-built micro-SD card for later analysis.

The programme has been developed through a participatory design process with 10 older adults and piloted in a home-deployment with 20 participants. The design and evaluation of this programme has taken place over a period of 16 months and is still ongoing. A more detailed report of the Alertness project can be found on our website [34].

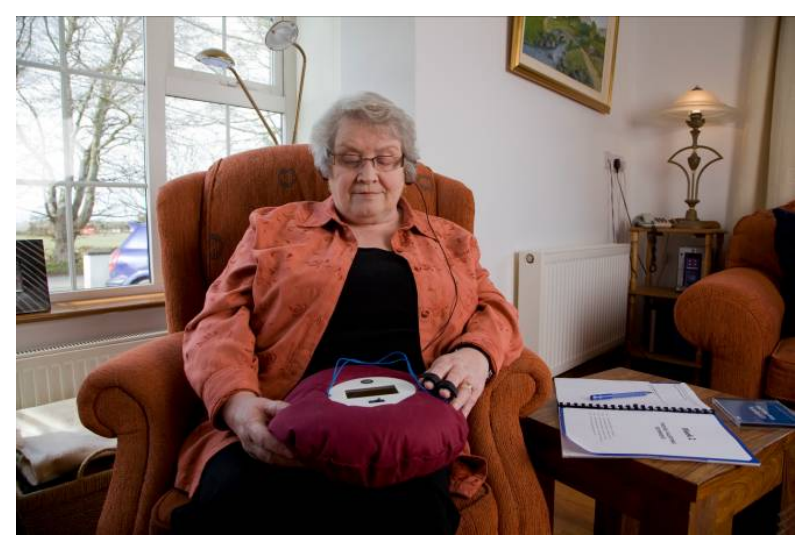

Figure 1 - Participant using the Alertness training programme

\section{Case Study Two: BASE - Balance and Strength Exercises}

The BASE (balance and strength exercises) in-home system is a hardware and software tailored exercise system, which is installed in the homes of older adults, who are at risk of falling. With BASE our aim is to focus specifically on improving older adults' lower limb strength and balance. To this end, we have integrated a specific, proven exercise regime for older adults - the Otago Exercise Programme is a strength and balance 
retraining program that has been shown to reduce falls in older adults by over a third [6]. Such programmes are typically administered by providing a person with a booklet of instructions on how to perform the exercises, and asking them to complete these exercises at home. BASE is intended for deployment into peoples' homes, to increase their independence and keep them living at home for a longer period of time. The benefit of a technology-based solution for the home is that it facilitates monitoring of the correct completion of each exercise, the quantity of exercises completed, and compliance to the exercise programme.

Prior to beginning the home deployment, the participant's physical capabilities are objectively recorded in a clinical setting by a physiotherapist, and their in-home exercise programme is tailored accordingly. During the home deployment, the physiotherapist can connect to the server and remotely observe and modify the participant's exercise program using the 'Physio Console' - an application developed to support the physiotherapist in remote monitoring of patients. The physiotherapist can monitor progress throughout the trial, modify the exercise programme, and provide feedback to participants where necessary.

The BASE in-home system consists of: 1 laptop; 1 interactive flash-based software application; 1 monitor; 1 remote control; 1 broadband connection; 2 adjustable ankle weights; 1 webcam; 3 tracking markers; 2 SHIMMER kinematic sensors [32]. The system is started and stopped by pressing a single large button located on the front of the laptop, and the flash software automatically loads on start-up. The flash software displays the instructional content (audio and video), feedback (real-time and trend) and navigational tools, which are prescribed to each individual user.

The flash application plays video instructions for each exercise before the user performs the exercises. The Otago program consists of warm-up, static sitting and standing exercises, and dynamic walking exercises. The BASE flash application provides real-time feedback on correctness of static and dynamic exercises using animations, repetition counters and audio prompts. Correctness of the static exercises is measured by tracking (with the webcam) the location of brightly coloured markers worn on the ankles and waist. Correctness of walking exercises is measured using SHIMMER motion sensors attached to the markers. Finally, the user controls the pace of the flash application and navigates between the menus using the BASE remote control - a small, lightweight control with five buttons (see graphic in Figure 2) that is attached to a string and can be easily worn around the participant's neck as they exercise.

To date, the design and evaluation of BASE has taken place over a period of one year. This has involved focus groups and in-home evaluations with a number of older adults. Further details on BASE, including the initial design process and usability evaluations can be found in [16]. 


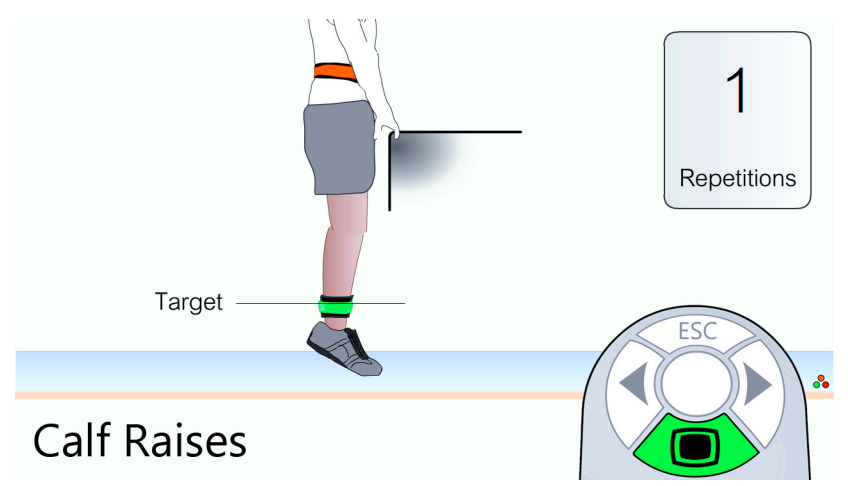

Fig. 2 Screenshot of visual feedback in BASE

Our recommendations, presented below, are divided into four sections representing the interrelated and dynamic life cycle of independent living technology development within TRIL: ethnographic inquiry; design; deployment and ongoing evaluation. It is important to note that they are based on the deployment of research projects. As such, the technologies represent prototypes rather than products and were deployed to answer research questions rather than as long term interventions. This paper does not claim to provide a definitive set of guidelines or recommendations that address all aspects of technology design and delivery for older adults; rather the aim of this paper is to reduce the gap in research on home deployments, by sharing our experiences of the complete life cycle of independent living technologies with other researchers in the field. A complete list of publications on TRIL's home-based research which have contributed to our recommendations can be found on the TRIL website. ${ }^{2}$

\section{AN ETHNOGRAPHIC PARTICIPANT-LED APPROACH}

The role of ethnographic enquiry in user-led design is well established. A user perspective opens up older persons' understanding of quality of life, well-being and ageing in place and ethnographic assumptions remind us that individual behaviours and beliefs may be shaped by individual and cohort life course experiences. These in turn are likely to shape expectations of existing and future technologies.

\section{Understand the importance of the user}

As we have written elsewhere [3], we started with the premise that for new technologies to be relevant to older persons' lives, we needed to both understand the user and understand the complexity of lives lived within the physical, emotional and social spaces that we refer to as 'home.' Moreover much of everyday life, daily routines, adaptation to change, notions of risk, all behaviours critical to developing useful technologies, are rarely reflected upon and may only be captured through observation.

With permissions, ethnographers attached to the TRIL team thus spent many months making repeat visits to the participants' home and accompanying them in their activities beyond the home. In this way we built case studies

\footnotetext{
${ }^{2}$ http://www.trilcentre.org/tril-research/publications.html
} 
that captured complex relations between how older people adapt, cope and adjust to everyday activity and orientation. Critically research questions based on actual need began to emerge. Ethnographic findings were interrogated by a multidisciplinary team including older adults. Moreover from within some pilot activity older adults also had input into the technology concept stage, supporting further development and refinement through workshop discussions, concept design, initial home deployment and ongoing evaluation within home and laboratory environments.

\section{Understand the importance of home}

A consistent finding of much ageing research, including that conducted by TRIL, is that older people want to stay in their own homes as they age. Individual attitudes and behaviours are likely to be partly influenced by a generational perspective, embedded in and shaped by, historical time and place [18]. Thus, whilst acknowledging a wide range of experiences, from within our TRIL cohort, a proportion may share similar home-based values. Some have lived for many years in the same house and may have experienced many life events while living there including marriage, raising families, recession and death. Such experiences may shape the meaning of home.

When designing for a home deployment, one must also consider the social and environmental factors surrounding the home - are there visitors, pets, care providers - and how do these engage with the older adult and the house? Will any of these factors impact on the technology that you are deploying? Our interactions suggest that for this cohort, home seems to be a place where others are welcomed in to share food and conversation and 'a bit of comfort'. Understanding the cultural context of home is pivotal to successful research engagement. The home is not simply four walls, but an eco-system consisting of the people who inhabit and interact with it. For example, we have had cases where a family pet, a grandchild or a home-help has triggered sensors. Speaking with family members' about their concerns is also critical. Indeed even when the older adult is willing to participate, family members may be concerned that there will be too many demands on their loved one. As we discuss later, we now provide more information to participants that they can share with family members.

\section{Assess attitudes towards technology interventions}

It is essential that those researching ageing understand the factors that might influence an older adult's acceptance and adoption of a technology, or lack thereof. Only then can we begin to design useful and usable technologies that might appeal to older adults and encourage motivation to use. A number of issues arose during our many interactions with older adults, the most common of which are discussed here.

Affordability is one of the most common questions raised by participants. There is not only the cost of the technology to consider but also any peripherals, such as a monthly cost for an internet connection. In deploying technology, it is necessary to consider the 'bigger picture'. While it might be beneficial for the purposes of research to use a high spec system, this will not be the most practical solution in the long term. Researchers should aim to use low-cost technology where possible. 
Another consideration is peoples' perceptions of their health. A number of times we heard older adults say that while they enjoyed using the technology "It's not for $m e$ ". According to the literature, perceived value or benefit is one of the main factors affecting an older adult's acceptance and adoption of technology [10]. We have also found that to be the case within TRIL. While most participants agree that such technology will benefit them at some point in the future, some of our more active participants feel they do not need assistance at their current point in life. This has a number of implications on design, such as keeping in mind that this notion of perceived benefit is a considerable factor of intrinsic motivation. It is thus important to identify user groups who will benefit from the technology and as such, who will want to use it. Participants of our BASE trials were very positive about using the technology, as each had suffered a fall in the past and were eager to undertake a programme that might prevent this from happening again.

\section{THE DESIGN PROCESS}

\section{Design for and with older adults}

There are a number of physical and cognitive factors associated with ageing that affect an older person's access to and usage of technology. Changes related to the 'normal' ageing process include physical decline, an increase in health issues, occasional forgetfulness, and reduced social engagement in the community. However, older adults are an extremely heterogeneous group, in which even age-matched adults are at differing levels of cognitive, physical and social decline due to the ageing process. Hawthorn [20] and Zajicek [38] both discuss the implications of ageing on interface design. Guidelines have also been published for designers developing websites for this age group [22, 39].

Older people also typically have less experience and familiarity with technology. As a result, this cohort has vastly different needs to younger people. The willingness of older adults to adopt technology is dependent on numerous factors, including familiarity [10,31], computer self-efficacy [10], relevance [31], and interest [35]. A comprehensive review of the factors affecting computer use amongst older adults can be found in [37].

Unfortunately, many designers typically have little understanding of the unique needs of older adults [28, 7]. Strategies are needed to engage older people in the design process so that designers can begin to 'get to know' their users. Within TRIL, each of our projects encompasses an iterative participatory design process, actively involving older participants in 'co-designing'. Designing technology in such a way does not only contribute to a higher probability of usable technology, but also fosters compliance in long term usage, by addressing specific needs. In the Alertness project this was achieved by having multiple interactions over time with participants, as part of the co-design process. This involved three home visits, a workshop and a pilot trial.

Ballegaard et al. [4] discuss the importance of participatory design methods in the development of healthcare technologies and emphasize the importance of fitting into the citizen's daily life and routine. They propose two guidelines for the design of technology for healthcare: design for continuity, by minimising the disruptive nature of new technology and design for understandability and learning. 
Older adults are usually unaware of the potential benefits of technology and as Zajicek [39] has pointed out, this can limit their ability to actively contribute to a discussion on technological requirements. We have found that using storyboards, scenarios and personas in focus groups can address this issue, by shifting the focus from the technology itself to what the technology can provide in terms of benefitting the individual. These techniques facilitate open discussion among participants and ultimately help the design team in identifying key features of the technology. It is worth acknowledging that while older adults typically have less experience with technology, they often have a wealth of other knowledge and experience. Our participants have included people from all walks of life and various professional backgrounds, from lawyers to professional athletes and many other highly educated and experienced older adults. Making use of this knowledge and experiences is critical in the design process to ensure participants are actively contributing, rather than passive test subjects.

We have also found it is necessary to educate older adults to be critical in their feedback. Older adults may often be over positive about their research involvement and experiences when what is really required is constructive criticism. Ethnographers, by spending extended periods of time with participants, can encourage such feedback. They 'set the stage' for critical feedback.

Through our experiences we have learned that it is important to design non-stigmatising technology targeted to a heterogeneous group of older people. By their nature, independent living technologies have a certain stigma attached to them, indicating that 'help' of some form is required. We have found that this stigma can be reduced through positive study names, which do not label the person as dependent. For example participants in the Alertness project, renamed the project from "Engineering Alertness", which implied a participant passitivity to "Training for focused living", a more empowering name, which emphasised the proactive role of the participant in training their alertness. The participants also identified unfamiliar technical jargon during the design process and suggested more common and intuitive alternatives. For example, 'calibrating' was changed to 'adjusting' and 'electrodes' became 'sensors'.

A further example of the importance of involving older adults in the design process became apparent when designing BASE. In deciding on the type of visual feedback to present to participants when performing exercises using BASE, we initially considered four options.

1. Our physiotherapist demonstrating the exercises on screen while the participant follows along. This option had a repetition counter that incremented with every repetition the participant completed. In this option there was no on-screen target (Figure 3 - top-left).

2. An abstract animation of the participant's markers on screen, with a target and a repetition counter. As the participant moved towards the target (the broken blue line), so did the animation of the markers (the thick orange line) - Figure 3 (top-right).

3. A higher fidelity 'matchstick man' animation with an on-screen target and repetition counter. As the participant moved, the 'matchstick man' mirrored this movement until the target was crossed (Figure 3 bottom-left). 
4. Real-time video feedback whereby the user could see themselves on-screen as they exercised. With this option participants could see their markers overlaid on their ankles and waist on the video feedback, as well as an overlaid target and repetition counter (Figure 3 - bottom-right).
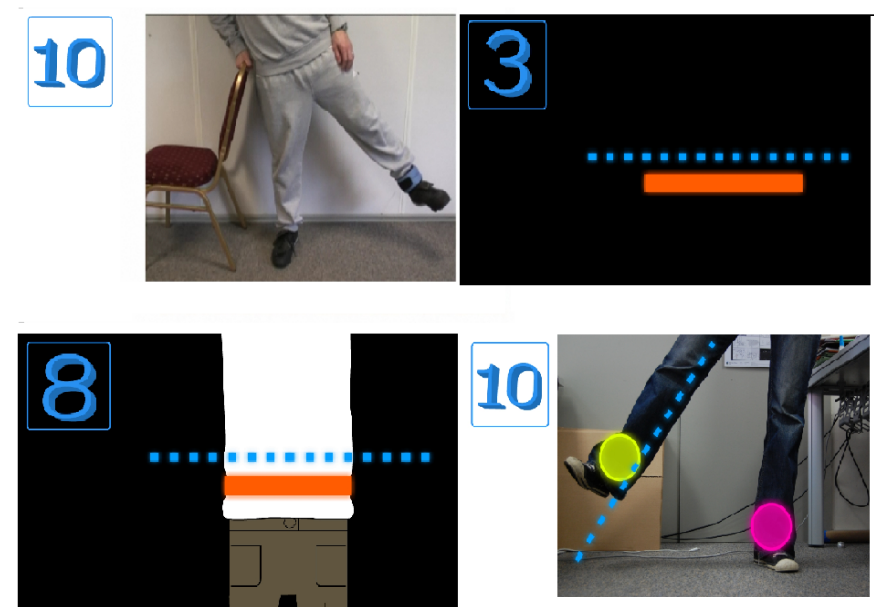

Figure 3. Types of visual feedback evaluated in the BASE design process - explained in details in points 1-4 above

The design team hypothesized that the fourth option, i.e. the real-time video feedback would be preferred by older adults. This was primarily because with this feedback it was easy to recover from errors, in that if the participant's marker was 'lost' during exercising, it was easy for the participant to notice this and correct it. This type of feedback was also a very natural mapping - i.e. the feedback represents exactly the participant and their actions. In order to test each of these methods of visual feedback, 6 participants performed a task that required them to move through five of the Otago exercises. Prior to beginning, the participant was asked to put on the markers, weights and SHIMMERs. For each exercise, the participant watched a demonstration video and then completed on average 10 repetitions with each feedback method. Having completed each exercise, the participant was asked for their opinion on which feedback option they preferred in terms of aesthetics as well as how effectively it helped them to complete their tasks.

In terms of preference, the high fidelity 'matchstick man' was chosen as first preference by each participant. The real-time video feedback was least favoured as participants felt 'foolish' or 'distracted' seeing themselves on screen. In addition, the surrounding environment was visible which further distracted participants. One participant stated "The matchstick man is really visible... you don't really want to see yourself". Another participant described it as "a good clear demonstration".

The matchstick man was further developed to appear more 'life-like' than that in Figure 3. Our current design can be seen in Figure 2. The matchstick man wears markers corresponding to those that the participant wears. If the system loses tracking of the participant's markers the matchstick man's markers will disappear. This in turn forces a re-calibration whereby the participant receives the appropriate instructions. We decided to represent the 
participant's on-screen target as a line. We initially used circles and squares as targets but this confused participants as to whether they needed to reach the outside of the shape, or the centre.

Thus, the design team's hypothesis that the video feedback would be preferred was not supported and was indeed the least preferred, demonstrating the importance in working with the user during the design process.

\section{Design with multidisciplinary team members}

The tenet of independent living technologies is that they address a clinical need, whether physical, social or cognitive. We are not developing technology for the sake of technology. As such, the design process must begin with expert input from health professionals and home care workers to determine clinical requirements. In designing BASE, for example, the process began with a geriatrician with expertise in falls in older adults outlining the desired outcomes of the project. These were then translated into design and engineering requirements, through a series of workshops with team members. At a certain point in the design phase of the project it was necessary to also bring a physiotherapist on board to ensure that the technology version of the exercise programme was being designed with safety considerations in mind.

Ethnographic data feeds our initial 'idea conception' and these are then expanded or refined with older adults through focus groups and workshops. Relaying the ethnographic data collected to the wider team is important at the beginning of the design process as well as throughout. Within TRIL we achieve this through holding 'data download' workshops with multidisciplinary team members. After each stage of design and evaluation with older participants, it is important to hold such a workshop to refine requirements and outline future developments.

Newell and Gregor [28] have argued that user-centred design (UCD) methodologies work well for 'typical' users, but not others (including older adults) given that such processes are typically carried out by designers who have limited knowledge of the characteristics of older people. In contrast, we have found that when the UCD process involves a multidisciplinary team including ethnographers, social and cognitive scientists as well as designers and engineers, the process is much more effective. In such scenarios, designers and engineers can learn a lot from the interaction between ethnographers (who typically moderate the process) and older adults.

Despite the benefits of a multidisciplinary team, there is also the danger of many varying opinions on how best to achieve a goal. It is important to remember that while each member of the team has an individual (research) goal, we all have a common mutual goal - to deploy technology that is both useful and usable. Finding commonalities helps to improve communication within the team [14]. Dawson [11] discusses the importance of providing the multidisciplinary team with guidance in interpreting ethnographic data. In the Alertness study we found that it is important for multidisciplinary team members to break down disciplinary walls and not to rigidly adhere to their own role.

\section{Integrate appropriate feedback into the technology design}

Feedback may have many purposes in a technology design. Appropriately designed feedback increases motivation to use the technology, enhances usability and provides progress updates to participants. In early focus group 
discussions for BASE, we asked participants about their previous experience with physiotherapy. We learned that adherence was generally very poor as participants were easily bored performing the same exercise repetitively and many reported ending their exercise programme when they themselves felt they were better. In hindsight however, many felt they stopped exercising too early and following a period of not exercising, found it difficult to remember the exercises they had been prescribed. Feedback within BASE thus played a crucial role in participant motivation and self-assurance. Real-time feedback during exercise informed participants whether they were exercising correctly. We also provided progress feedback and highlighted the importance of adherence throughout the technology programme. Any reinforcement should be positive and encouraging, to ensure participants remain motivated to continue.

In terms of improving usability, feedback should provide confirmation that the technology understands input from the participant as well as delivering prompts for using the technology and for recovering from errors, whether user or system based. For example, through observation and feedback in BASE design sessions with older adults we determined that prompts (both visual and audio) would be necessary to aid the participant in moving between menu items and exercises, as each participant found this particularly difficult. Audio navigation prompts include, for example, 'Press GO to move on', 'Remember you can pause at any time'. The visual prompt can be seen in Figures 2 and 4. It is an animated graphic of the remote control. For each current state the participant is in, there are one or more new states where the participant can move on to. For example, in Figure 2, the participant can pause during exercising. This provides them with the option to resume exercising or to skip to the next exercise (if they find they cannot reach their target). In Figure 4, the participant can select the centre button to begin their exercise program. They can also use the right navigation button to move onto other menu options, i.e. to view their exercise records or to shut down the system. Within this menu, the currently selected menu item is enlarged. Furthermore, as the user moves to each option, an audio instruction is output. For example, the Shut Down option outputs 'Turn off the system', thus compensating for the visually impaired.

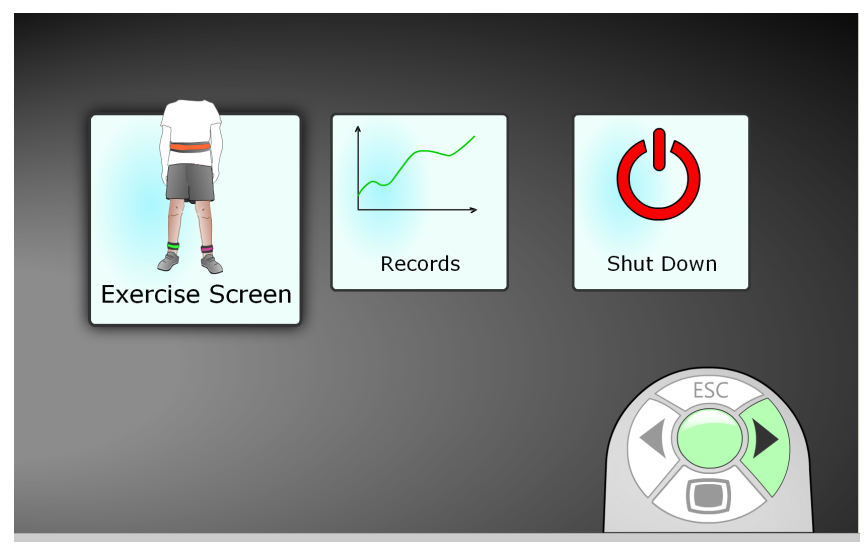

Figure 4. Navigation assistance in BASE 
The remote control graphic appears on each screen, prompting the user as to how they can move on, when they can pause etc. If the system is waiting for user input before moving on, the appropriate button will slowly flash, prompting the user for input. This navigation aid was tested preliminarily during user testing in older adults' homes. We observed no issues with participants following the on-screen prompts. However, participants said they would like to see an instructional video on how to use the remote control functions, and what each button represents. This has been implemented and is played at the start of the exercise program.

Multimodal interaction, in terms of both input and output, is likely more beneficial for this cohort than unimodal interaction. Not only does multimodal feedback counteract potential sensory deficiencies but it can be essential for certain technologies that require focus on something other than the technology, for example a participant concentrating on performing a motor task as in BASE.

In the Alertness project, we utilised biofeedback to support independent training of the self-alerting technique. By observing the effect of the self-alerting technique on GSR readings on the device's screen, the participants were given an insight into whether they were conducting the technique correctly. Observing an increase in GSR reading on the device after using the self-alerting technique also motivated participants to continue practicing the technique in order to get stronger responses in their GSR readings.

\section{Design for enjoyment}

Enjoyment is considered a crucial factor of intrinsic motivation. To be effective interventions, independent living technologies require long term, continued usage. However, in the context of home deployments, this can be very difficult to achieve, as the participant is left to use the device as much or as little as they like. Furthermore, any initial motivation to 'help the researcher' is likely to fade in a long deployment. As such, making the technology and the study enticing is a critical design requirement to motivate usage. Within the Alertness project, we used ethnographic analysis of a previous Alertness deployment to find and possibly increase the enjoyable elements. For example, a previous deployment involved sending participants a kit through the post with a 'do not open until instructed' note on the envelope. This motivated people to participate by triggering curiosity. With our most recent Alertness deployment we extended this by having 2 'do not open until instructed' boxes, one to be opened in the first week and one in the third week (which held the device). Again, this added an element of fun, curiosity and enjoyment to the trial.

Indeed, if the technology requires regular interaction, the technology itself must be enjoyable. Developing on the idea from the Alertness project, opening up further functionality when then participant has reached a certain level of usage can ensure continued enjoyment and 'novelty'. In a previous TRIL project examining the use of communication technology to connect older people, we had limited the number of functions to prevent the technology from being complex [17]. However, we realised that after a short period of usage, participants became very familiar with these functions and began to require additional ones to maintain their interest in the technology. Thus, in BASE, we plan to 'open' new exercises and functionality once participants have become comfortable with existing ones. 


\section{Balance between usability and functionality}

Corry, Cramp and Aalokke observed that "It is not enough to make a beautiful GUI on a laptop" in [9] and it has also been our experience within TRIL. When evaluating an early, laptop-based version of the biofeedback technology in the Alertness project, participants were anxious when operating the laptop without assistance, afraid to 'press the wrong button'. This anxiety was largely reduced by removing all the unused features that the laptopbased solution offered. Apart from an increase in reported ease of use of the 'stripped back' technology, a clear indicator of increasing engagement through reducing complexity was the fact that the undirected feedback of the participants was more focused on interpreting the biofeedback signal, and not on the device through which the biofeedback was mediated.

While it makes sense to remove some functionality, there has to be a balance in that the participant has to be aware of potential ways of restarting the system following a crash or a breakdown. For example, we've found that you can't install a wireless router and simply tell the participant that they don't need to know what it is. Understanding of all components that are being deployed is important. However, participants don't need to know all the workings of the system as this will result in information overload. BASE uses an auto launch script that launches the application or shuts it down, once the participant presses a custom made 'start and stop' button that is placed beside the monitor. This removes the complexity of using a mouse and keyboard to start and shut down the system.

\section{Ensure technology and related kit easily integrate into peoples' homes}

Our goal within TRIL is to deploy technology into people's existing homes. As such, we must fit the technology into the participants' lives, not make their lives fit in with the technology. Technology should be unobtrusive, aesthetically pleasing and shouldn't appear stigmatising. This means it is essential not to recreate a lab or clinical setting in the home. This should be remembered during the design process. For example, the Alertness cushion is designed around someone sitting on a couch or armchair. Other designs were also mocked up and evaluated, one of which was a desk-model. While this seemed a realistic design option when producing form factors (which was done in an office setting), during evaluations of the mockup in older adults' homes, desk models were quickly dismissed for two reasons - the lack of desks in peoples' homes and the negative associations such a 'desk' model had with school desks.

Similarly within BASE a number of revisions were made to the balance support, which was an essential component of the kit to ensure the participant's safety while exercising. While in a clinical setting balance bars would be used, this solution was highly unsuitable for a home environment. Consultations between the design team and clinicians resulted in the decision to use a chair, which could be modified to act as a support. However, when we visited participants' homes to test the feasibility of the chair and the related kit (consisting of a small shelving unit with a monitor and the laptop) a number of issues quickly became apparent. The chair often didn't fit into a room. Participants commented that it would be difficult to tidy it away and that they wouldn't want it on

display if they had guests over. As the chair was modified to act as a balance, some participants found it 
stigmatising. Furthermore, in-home observation by the physiotherapist on the team deemed the chair unsafe as the participant was required to move it into different positions for different exercises. We thus decided to search the house for a safe place to hold onto when exercising. This is typically how the non-technological version of this exercise programme is undertaken - known as 'kitchen sink' exercising, participants are asked to use their sink as a support. This increased the complexity of the design requirements for the deployment, as the kit had to be flexible enough to be located in a different area in each individual's house. However, the primary concern was for the participant's safety and their satisfaction with the placement of the kit into their home.

Evaluating the home prior to deployment of technology is an essential step in ensuring the technology will easily assimilate into the participant's home and their daily living. This is primarily a design-led approach whereby the living space is mapped out to ascertain what spaces are used for certain activities and to assist in identifying optimal locations for technology placement considering practicalities such as access to power sources. We have discussed this further in previous work [15].

\section{Allow for flexibility in usage}

Similar to assimilating unobtrusively into a person's home, technology should not disrupt their day-to-day living. The majority of the older people we have worked with have quite busy lives that researchers must fit the technology around. It is the participant who should control when they use the technology. During the Alertness project we asked participants to 'work' 5 days a week for 4 weeks. Each session typically lasted 15-30 minutes and participants were free to choose the time that suited them. Explaining this in our discussions with potential participants prior to deployment also helped with recruitment, as people don't like to commit to something that is intense or strict.

The Alertness training programme was also developed to be flexible in where the kit could be used, whether in the home or outside of the home. This was made possible by integrating light-weight, portable components and making the cushion battery powered.

Similarly in BASE, participants were told that to benefit from such an exercise programme, they would need to use the system 3 days a week and that they shouldn't exercise two days in a row. Otherwise, it was left to the participant to decide when they used the programme.

\section{Ensure technology is usable prior to deployment}

While it has been our experience that many usability issues only arise during a home deployment and longer period of use, it is important to iteratively evaluate technology in a number of 'once-off' sessions to remove as many issues as possible prior to deployment. This is especially important when deploying technology to vulnerable groups. Through our deployments we have learned:

- Technology will simply not be used if participants have any difficulty in using it.

- Unusable technology can have negative effects on older adults' motivation to use any form of technology. 
- Many participants feel like they are doing something wrong if the technology is not operating as expected.

Given that technology is often new and unfamiliar to the majority of older adults, it is essential that their initial experiences are positive, as the potential negative effects of the introduction of inappropriate technology to this group are numerous, particularly when participants are frail and lonely [13].

\section{DEPLOYMENT TO THE HOME}

Independent living technologies will not make a positive impact on the ageing process unless they are actually deployed to the home. One of the major goals of our work has been deployment to the home, for a number of reasons. It is not only the individual to take into account but also his/her context of use. Home deployments allow us to study adherence to usage and assess motivation to use such technology, all of which can be fed into the design of an end product. Furthermore, given the goal of independent living technologies are to support the person in staying in the place of their choice as they age, it is imperative that they are evaluated in their intended place of use.

The complete process of home deployments, from installation, to usage by the participant to the final removal of the technology must not be disruptive or too time-consuming as this can have dramatic effects on the older population. Researchers should therefore always keep in mind that technology should assist day-to-day living, not increase its complexity. The following sections address some ways to ensure disruption is minimal.

\section{Flexibility in installing and uninstalling technology}

We found that we needed to be flexible and fit the technology deployment, data collection and deinstallation visits around participants' lives; to explain the purpose and function of the technology deployed; not to assume that both written and verbal information at the beginning of deployment would suffice. Often queries and curiosities would arise during deployment, for example explaining that motion sensors only record movement, not sound or vision may initially be understood. However family and friends visiting the home and becoming aware of such sensors, positioned over doorways for example, may start to question their function. Ongoing visits with time for tea and chat as well as data collection thus need to be built into research timescales. Moreover we also found that sharing with the participants the engineering data collected by such technology, further allayed concerns about privacy [2].

There was also a need to give participants time to consider their involvement. Some were familiar with more traditional research data collection such as taking part in focus groups, being interviewed or completing a survey. Committing to taking part in virtual social networking or a regular programme of ICT supported balance and strength exercises was in most cases, a new undertaking. Our recruitment strategy was two staged. Participants were initially recruited onto the wider TRIL programme through a number of referral points that included GP and self referral. During a research assessment within the TRIL Centre, the TRIL nurse manager would elicit interest in potential involvement in particular technology home deployment, giving written and verbal information. An 
ethnographer would follow up this initial approach with a phone call and if appropriate arrange a meet and greet home visit. At all stages, the participant was free to withdraw. What we found is that tentative curiosity often became enjoyably commitment. We have written more extensively about this elsewhere [2].

\section{Provide a paper-based information booklet}

We learned from our experiences with early projects in the first year of TRIL that providing an information booklet to participants is of immense benefit for a number of reasons. First, it can act as a help manual, providing a description of how to use the system. It should also provide a description of the research and a clear outline of the data being collected. In an early project within the Falls Prevention strand, a number of motion and velocity sensors were placed in older adults' homes over thresholds such as doors, and along walkways. Many participants were concerned that the sensors were cameras that were taking pictures as they moved around. This caused particular concern for sensors on bedroom and bathroom doors. In response to this, booklets were produced that showed, visually, what the researchers saw from the sensor output. When participants realised it was a series of numbers, their minds were put at ease.

Information booklets should also include an explanation of who the researchers are, along with photos and phone numbers in case the participant has any questions during the trial. Including researcher photos reminds the participant of who to expect at their home when the time comes for a visit from the researcher. This information also helps to put family members at ease as to what the research is about and why their relative is taking part. Furthermore, we have found that participants typically like to show these booklets to family and friends, as they have a feeling of importance in being chosen to take part in the study.

Booklets should also contain a calendar of 'events' during the research project, for example what dates the participant can expect a visit, whether they have appointments with a clinician or physiotherapist before or after the trial, as was the case with BASE. We have received very positive feedback on our information booklets from participants.

\section{Be prepared to deal with technical issues and breakdowns}

Homes are uncontrolled environments and any number of issues may arise that require planning and integration into the research plan. Through our experiences we have identified some ways of limiting potential issues. Others, however, require researcher input.

There are three areas here that we consider. Firstly, many independent living technologies are deployed with sensors which have limited battery life. To avoid issues of 'dead' batteries, participants should be given control of battery maintenance. We have found this requires not only training during deployment, but also reminders through the technology. In BASE, when participants finish their exercise programme, a video is played on screen as a reminder to charge the SHIMMER sensors, indicating to participants how they should be placed into the battery dock. 
Secondly, software bugs can typically be fixed by researchers remotely, by logging into the participant's system. The participant needs to be notified in advance of this and a thorough explanation provided of what this entails. Finally, hardware issues will most likely require a visit from a technician, which again should be discussed with the participant in advance. When making such visits, it is important to enter and exit the home efficiently and to facilitate this, systems should be swappable.

Participants should always be provided with a help line phone number which they can call 24 hours. This is important as if participants have an issue they can become quite nervous as they're usually not sure what is happening or what the outcome of the problem might be. We often got calls outside of normal working hours. Most times the participant simply needed reassurance that they hadn't 'broken' the technology or that the issue wasn't critical. If there were further concerns, we offered to call out during working hours. Promptly dealing with any participant issues is extremely important. While a participant would 'forgive' a failing technology, they are rightfully not as forgiving if researchers fail to deal with their concerns.

\section{EVALUATION AND EXIT FROM THE HOME}

Evaluation of independent living technologies should be a continuous process occurring at all stages of the life cycle of the technology. Prior to home deployment this includes bench testing with multidisciplinary team members to eliminate major issues in addition to focus groups and lab-based usability testing with older people. If the technology intervention is particularly complex it is advisable to run pilot home trials, testing with a subset of the target group for a short period of time. This section focuses on our lessons learned in evaluating independent living technologies in addition to outlining effective exit strategies when a deployment has come to an end.

\section{Make use of interaction logging}

To date, our deployments have typically lasted between 6 and 12 weeks. To gain an objective view of usage levels, for example what features are used frequently or sporadically, as well as usability issues such as what aspects of the system might have caused confusion or errors, interaction logging is essential. This must be approved by an ethics board, as well as by the individual participant.

Researcher analysis of interaction logs can also be useful in providing feedback to participants during 'exit' interviews (i.e. at the end of the deployment). A number of our participants have been curious about 'how they got on' and requested such feedback.

\section{Use an evaluation protocol}

Exit interview sessions have to be carefully planned and well organised so as not to take up too much of the participant's time. As our deployments have been research related and involve a multidisciplinary team, there are a number of stakeholders interested in evaluating different aspects of the deployment. However, when participants are being asked many questions, or asked to interact with the device so we can observe, this can quickly become tiring. Developing an evaluation protocol in advance and having 'dry-runs' of this with team members can make the evaluation run more smoothly. The evaluation protocol should include the specific goals of the evaluation; the 
researchers involved; the sequencing and timing of events, such as usability observation, interviews etc.; a list of tasks the participant will be asked to complete; a list of questions they will be asked; methods of recording data and the equipment needed (e.g. video camera, audio recorder). Time should also be factored in for chats, a cup of tea and questions from the participant. Having a well thought-out schedule provides a sense of togetherness to the participants, making them feel at ease.

\section{Evaluate with drop-outs}

While we endeavor to fully prepare participants prior to deployment of technology about the nature of the study and what exactly they should expect, there have been times when some participants have decided to drop out of the study, either during or prior to deployment. Evaluating with these participants can provide invaluable insight into how to improve our recruitment and retention strategies as well as into design, as such participants often have concerns surrounding privacy, stigmatisation or otherwise.

\section{Plan effective 'Exit' strategies}

Exit planning is shared with the participant from the start of their involvement. As we have said, flexibility is a key component of retention, so from the start, we outline our participant led approach, all planned stages of the research and this includes our exit phase.

This exit phase includes de-installation and an evaluative exit interview with the participant. We also build in time to talk and express our gratitude for their participation and we often return the data, creating simple house plans for example, or images of the technology and the engineering data. In the Alertness deployment we scanned the training guides, returning the original copies to participants. Participants like to share these with family. They also act as reminders of their participation, as one participant commented ". . . will remind me of the time when I was

\section{'Trilling'!"}

Within a few days of the exit home visit, follow up phone calls are made to ensure that there are no loose ends and also to give the participant an opportunity to discuss any outstanding issues. In relation to research closure, and like others we were mindful of the fine line between researcher and friend particularly for older adults who may have expressed loneliness [25]. However we suggest that discussing and outlining a clear exit strategy from the beginning, with a proviso of voluntary early withdrawal, building in time for reflective discussion during the exit home visit, preparing a 'research memorabilia' and building in a follow up phone call, create an agreed and expected closure and completion framework.

\section{DISCUSSION}

Independent living technologies present huge potential to provide a better quality of life for older people, helping them to remain independent in the place of their choice as they age. However, bringing research of such technologies out of the lab and into the home for long periods of time still remains a relatively rare occurrence. To be successful in the short term, technologies deployed to homes must collect high quality context data, suitable for clinical research and useful for feedback to the older adult. To be successful in the long term, such technologies 
should ultimately help older adults to age-in-place, ideally in their own homes, supporting them in living healthy independent lives. We argue that research needs to become more applied, if such technologies are to make any real positive impact on the well-being of older adults.

Thus, we have presented here our experiences and lessons learned in working with over 200 older adults in their homes. From these experiences, we have outlined a series of recommendations to act as a guide to other researchers working in the field, particularly younger early-stage researchers who most typically carry out this type of work. As we realized during our research, not everything can be learned through a book or lectures. There are some things that can only be learned through experience. When working with older adults however, having an insight into others' practical experiences can be hugely beneficial not only for the researcher but for the older participants as their experience can be enhanced if the recommendations we have presented are followed. We want to highlight the importance of a cyclical, iterative process, at each stage of which the older adult plays an integral role.

Unfortunately, this paper cannot accommodate all of the experiences we would like to share, but we have presented here what we feel are the most important issues. As we continue to work with older people we hope to expand this work into a more detailed set of guidelines. Ultimately, we hope our recommendations, summarized below, will act as a practical guide for others.

\section{SUMMARY}

\begin{tabular}{|l|l|}
\hline Theme & Recommendation \\
\hline Ethnographic, & Understand the importance of the user. \\
\hline & Understand the importance of home. \\
\hline & Assess attitudes towards technology interventions. \\
\hline The Design Process & Design for and with older adults. \\
\hline & Design with multidisciplinary team members. \\
\hline & Integrate appropriate feedback into technology design. \\
\hline & Design for enjoyment. \\
\hline & Balance between usability and functionality. \\
\hline & Ensure technology and related kit easily integrate into peoples' homes. \\
\hline & Allow for flexibility in usage. \\
\hline Deployment to the home & Ensure flexibility in installing and uninstalling technology. \\
\hline & Provide a paper-based information booklet. \\
\hline & Be prepared to deal with technical issues and breakdowns. \\
\hline
\end{tabular}




\begin{tabular}{|l|l|}
\hline $\begin{array}{l}\text { Evaluation and exit } \\
\text { from the home }\end{array}$ & Make use of interaction logging. \\
\hline & Use an evaluation protocol. \\
\hline & Evaluate with drop-outs. \\
\hline & Plan effective 'Exit' strategies. \\
\hline
\end{tabular}

\section{REFERENCES}

1. Alm, N., Gregor, P. and Newell, A.F. Older people and information technology are ideal partners. In International Conference for Universal Design, 2002.

2. Bailey, C. and Buckley, V. Recruiting and retaining older persons within a home based pilot study using movement sensors. In Health and Social Care in the Community, (2010), in press.

3. Bailey, C. and Sheehan, C. Technology, older persons' perspectives and the anthropological lens. In ALTER, European Journal of Disability, 3, Elsevier, (2009), 96-109.

4. Ballegaard, S.A., Hansen, T.R. and Kyng, M. Healthcare in everyday life - designing healthcare services for daily life. In CHI 2008, ACM press (2008), 1807-1816.

5. Bieber, G., Koldrack, P., Sablowski, C., Peter, C. and Urban, B. Mobile physical activity recognition of stand-up and sitdown transitions for user behaviour analysis. In PETRA Workshop on Affect and Behaviour Related Assistance for the Elderly, (2010).

6. Campbell, A.J., Robertson, M.C., Gardner, M.M. et al. Randomised control trial of a general practice programme of home based exercise to prevent falls in elderly women. In BMJ 315, (1997), pp. 1065-1069.

7. Carmichael, A., Newell, A.F. and Morgan, M. The efficacy of narrative video for raising awareness in ICT designers about older users' requirements. In Interacting with Computers, 19, Elsevier, (2007), pp. 587-596.

8. Carmichael, A., Rice, M., MacMillan, F. and Kirk, A. Investigating a DTV-based physical activity application to facilitate well-being in older adults. In BCS HCI 2010, (2010), in press.

9. Corry, A.V., Kramp, G. and Aalokke, S. Balancing user control. In Designing for Palpability: Workshop at Pervasive 2007, ACM press (2007).

10.Czaja, S., Charness, N., Fisk, D., Hertzog, C., Nair, A.N., Rogers, W.A. and Sharit, J. Factors predicting the user of technology: Findings from the Center for Research and Education on Aging and Technology. In Psychol Aging, 21 (2), (2006), pp. 333-352.

11.Dawson, M. Anthropology and industrial design: a voice from the front lines. In Squires, S. and Byrne, B (Eds.) The Collaboration of Anthropologists and Designers in the Product Development Industry, Bergin \& Garvey, (2002).

12.Demeris, G., Rantz, M., Aud, M., Marek, K., Tyrer, H., Skubic, M. and Hussam, A. Older adults' attitudes towards and perceptions of 'smart home' technologies: A pilot study. In Medical Information, 29, (2), (2004), pp. 87-94.

13. Dickinson, A., Goodman, J., Syme, A., Eisma, R., Tiwari, L., Mival, O. and Newell, A. Domesticating technology. Inhome requirements gathering with frail older people. In $10^{\text {th }}$ International Conference on Human Computer Interaction, (2003), pp. 827-831. 
14. Dourish, P. Implications for design. In CHI '06, (2006), pp. 541-550.

15. Doyle, J., Bailey, C., Dromey, B. Experiences of in-home evaluation of independent living technologies with older adults. In I-HCI, $3^{\text {rd }}$ Irish Conference on Human Computer Interaction, 2009.

16. Doyle, J., Bailey, C., Dromey, B. and Ni Scanaill, C. BASE - An interactive technology solution to deliver balance and strength exercises to older adults. In Pervasive Computing Technologies for Healthcare (Pervasive Health 2010), IEEE, (2010).

17. Doyle, J., Skrba, Z., McDonnell, R. and Arent, B. Designing a touch screen communication device to support social interaction amongst older adults. In BCS HCI 2010, (2010), in press.

18. Elder, G.H. and Johnson, M.K. The life course and aging: challenges, lessons and new directions. In R.A. Setterson, Jr. (Ed.) Invitation to the life course: toward new understanding of later life, Part II. Amityville, NY: Baywood, pp. 49-81.

19. Gardner, D. and Helmes, E. Locus of control and self-directed learning as predictors of well-being in the elderly. In AustPsychol, 34, (2), (1999).

20. Hawthorn, D. Possible implications of aging for interface designers. In Interacting with Computers, 12 Elsevier, (2000), 507-528.

21. Hayutin, A.M. Graying of the Global Population. In Public Policy and Aging Report, 17, (2007), pp. 12-17.

22. Holt, B. and Morrell, R. Guidelines for website design for older adults: the ultimate influence of cognitive factors. In Older Adults, Health Information and the World Wide Web, (2002), pp. 109-132.

23. Jones, C., Winegarden, C. and Rogers, W. Supporting healthy aging with new technologies. In ACM Interactions, 16 (4), (2009), pp. 48-51.

24. Kinsella, K., Phillips, D. Global Aging: The Challenge of Success, 60 (1) Population Reference Bureau, (2005).

25.Locher, J., Bronstein, J., Robinson, O., Williams, C. and Ritchie, C. Ethical issues involving research conducted with homebound older adults. In The Gerontologist, 46 (2), (2006), pp. 160-164.

26.Lorenz, A., Mielke, D., Oppermann, R. and Zahl, L. Personalized mobile health monitoring for elderly. In $9^{\text {th }}$ International Conference on Human Computer Interaction with Mobile Devices and Services, (2007), pp. 297-304.

27. Melenhorst, A-S., Rogers, W. and Caylor, E. The use of communication technologies by older adults: exploring the benefits from the users perspective. In Human Factors and Ergonomics Society $45^{\text {th }}$ Annual Meeting, (2001).

28. Newell, A., Gregor, P. Human computer interfaces for people with disabilities. In Handbook of Human Computer Interaction, Helander, M. (Ed.), Elsevier, (1997), 813-824.

29. Rodriguez-Ascaso, A., von Niman, B., Brown, S. and Sund, T. Human factors: user experience design guidelines for telecare services. In Human Computer Interaction, Part IV, HCII 2007, Springer LNCS (2007), 413-417.

30. Sainz-Salces, F., Baskett, M., Llewelyn-Jones, D. and England, D. Ambient interfaces for elderly people at home. In Ambient Intelligence in Everyday Life, (2006), pp. 256-284.

31. Selwyn, N., Gorard, S., Furlong, J. and Madden, L. Older adults' use of information and communication technology in everyday life. In Ageing \& Society, 23, (2003), pp. 561-582. 
32. SHIMMER Research. (http://shimmer-research.com)

33.Sokoler, T. and Svensson, M.S. Embracing ambiguity in the design of non-stigmatizing digital technology for social interaction among senior citizens. In Behaviour and Information Technology, 26 (4), (2007), pp. 297-307.

34. TRIL Centre Alertness home deployment. Available at http://bit.ly/9KNwUH

35. Umemuro, H. Computer attitudes, cognitive abilities and technology usage among Japanese older adults. In Gerontechnology, 3 (2), (2004), pp. 64-76.

36. United Nations, World Population Ageing, 2007.

37. Wagner, N., Hassanein, K. and Head, M. Computer use by older adults: A multidisciplinary review. In Computers in Human Behaviour, 26, Elsevier, (2010), pp. 870-882.

38.Zajicek, M. Interface design for older adults. In Workshop on Universal Accessibility of Ubiquitous Computing, (2001), 60-65.

39.Zajicek, M. Aspects of HCI research for older people. In Universal Access in the Information Society, 5 (3), Springer, (2006), 279-286.

40.Zaphiris, P., Kurniawan, S., Ghiawadwala, M. A systematic approach to the development of research-based web design guidelines for older people. In Universal Access in the Information Society, 6 (1), (2007), pp. 59-75. 